\title{
Humanistic and Economic Burden of Geographic Atrophy: A Systematic Literature Review
}

\author{
Sujata P Sarda' \\ Anne Heyes ${ }^{2}$ \\ Meryem Bektas $\mathbb{D}^{2}$ \\ Tanvee Thakur ${ }^{2}$ \\ Wendy Chao (iD) \\ Michele Intorcia' \\ Samantha Wronski \\ Daniel L Jones'
}

'Apellis Pharmaceuticals, Waltham, MA, 0245I, USA; ${ }^{2}$ RTI Health Solutions,

Research Triangle Park, NC, 27709, USA
Purpose: Geographic atrophy (GA), the advanced form of dry age-related macular degeneration, can result in irreversible blindness over time. We performed a systematic literature review to assess the humanistic and economic burden of GA.

Methods: Predefined search terms were used to identify studies in PubMed, Embase, and Cochrane Library; conference abstracts also were searched.

Results: Of 1111 unique studies identified, 25 studies on humanistic burden, 4 on economic burden, and 3 on both humanistic and economic burden of GA were included. Vision-related functioning and health-related quality of life (HRQOL) are poor in patients with GA. HRQOL is commonly measured using the 25-item National Eye Institute Visual Function Questionnaire (NEI VFQ-25); patients with GA have significantly lower composite and subscale scores for near activities, distance activities, dependency, driving, social functioning, mental health, role difficulties, color vision, and peripheral vision than individuals without GA. Driving is a particular concern, and inability to drive affects dependency. Vision-related quality of life (VRQOL) declines as GA progresses. While we identified only 7 reports describing the economic burden of GA, its direct costs may be substantial. In a US study, mean cost to the payer per patient with GA was $\$ 11,533$ in the year after diagnosis. A multinational study estimated annualized total direct costs of $€ 1772$ per patient with GA, mainly driven by diagnostic tests and procedures (€1071). Patients with GA are at increased risk of falls and fractures, potentially increasing direct costs. Only one study evaluated indirect costs, estimating $\sim \$ 24.4$ billion in yearly lost wages among people with severe vision loss due to GA or drusen $\geq 125 \mu \mathrm{m}$.

Conclusion: GA represents a significant humanistic burden. Evidence on the economic impact of GA is limited; characterizing the economic burden of GA requires further research. Interventions that reduce GA-related disability may improve HRQOL and reduce indirect costs.

Keywords: dry age-related macular degeneration, health-related quality of life, visionrelated quality of life, costs

\section{Introduction}

Geographic atrophy (GA) is generally defined as the advanced or late stage of dry age-related macular degeneration (AMD) with a circular area of atrophy of $175 \mu \mathrm{m}$ or more. ${ }^{1,2}$ However, minimum sizes to define atrophic patches vary. ${ }^{2}$ In contrast to neovascular AMD, which is characterized by acute vision loss, GA is a progressive disease that can lead to irreversible blindness over time. ${ }^{1}$ GA interferes with daily activities such as driving, reading, writing, and recognizing faces, and in turn, negatively affects quality of life, potentially impairing mobility and independence. $^{3-5}$ The incidence of GA is not well understood, as it is likely
Correspondence: Daniel L Jones

Tel + I 6179775700

Email daniel.jones@apellis.com 
underdiagnosed and underreported. A meta-analysis of US populations of white European ancestry reported incidence rates of 1.9 per 1000 person-years (95\% confidence interval [CI], 1.3-2.8) for adults aged 50-97 years and 4.3 per 1000 person-years (95\% CI, 2.9-6.2) for adults aged $\geq 65$ years. ${ }^{6}$ An estimated $12-20 \%$ of patients with GA have severe vision loss, ${ }^{7}$ potentially requiring caregiver help with transportation and activities of daily living.

Several nonmodifiable and modifiable risk factors are associated with development of GA and risk of disease progression. $^{1,8,9}$ A number of genetic polymorphisms of the complement components and its regulators have been shown to be risk factors for developing AMD. ${ }^{8,9}$ Age and family history are significant risk factors for developing GA; cigarette smoking, diet pattern, physical activity level, and health conditions including hypertension, hyperlipidemia, obesity, and other ophthalmic comorbidities (eg, diabetic retinopathy and glaucoma) also have been reported to correlate with GA onset. $^{1,4,8}$ No effective treatments are indicated to prevent or slow the progression of GA; consequently, it has a poor prognosis. ${ }^{10}$ Currently, the only potential approach to preventing GA is avoiding modifiable risk factors such as smoking and maintaining a healthy lifestyle. ${ }^{1,8}$

To describe the burden of illness of GA and identify evidence gaps in the literature, we conducted a systematic literature review focusing on the humanistic and economic burden of disease and health-care resource use. The clinical burden of GA was also evaluated in this review but is not reported here.

\section{Materials and Methods}

A systematic literature review was conducted to identify studies indexed in PubMed, Embase, EconLit, and Cochrane Library. Abstracts from relevant congresses (American Academy of Ophthalmology, Association for Research in Vision and Ophthalmology, and European Association for Vision and Eye Research) were searched for 2019-2021 to identify recent information not yet indexed in medical literature databases. Additional data sources included bibliographies of included systematic reviews and meta-analyses, the grey literature, and clinical trials for emerging therapeutic agents for GA via ClinicalTrials.gov using the search term "geographic atrophy." Search terms included combinations of free text and Medical Subject Headings or Emtree subject headings. Two sets of searches were conducted: the first searches identified studies published from January 1, 2010-
August 11, 2020. Because very little evidence on the humanistic or economic burden of GA during this window was identified, a second set of searches identified studies on the humanistic or economic burden of GA published from January 1, 2005-January 22, 2021. Studies were chosen in accordance with predefined eligibility criteria; additional details regarding the search methodology are presented in Tables S1-S4, Supplemental Appendix A. Relevant data were extracted directly from the identified studies. Costs presented in the included studies were converted, as applicable, to US dollars (USD) in the reported cost year; all costs were then inflated to 2020 USD. Exchange rates and inflation factors were taken from the Organization of Economic Co-Operation and Development website; ${ }^{11}$ additional details are presented in Tables B1 and B2, Supplemental Appendix B. No additional analyses across studies were produced.

\section{Results \\ Search Results}

Of 1111 unique studies identified, 142 were included in the broader systematic literature review (Figure C1, Supplemental Appendix C). Of these, 32 studies were included in the review: 25 on the humanistic burden, 4 on the economic burden, and 3 on both humanistic and economic burdens.

\section{Humanistic Burden}

Table 1 summarizes studies describing the humanistic burden of GA. Most used the 25-item National Eye Institute Visual Function Questionnaire (NEI VFQ-25) to describe the burden of GA.

\section{Measurement of Quality of Life and Reading Function in GA}

Three patient-reported outcome measures are commonly used in prospective trials of GA (see Supplemental Appendix D). Briefly, the validated patient-reported NEI VFQ-25 is commonly included in studies in AMD to evaluate disease burden and has undergone preliminary validation in patients with GA, ${ }^{12}$ although Rasch analysis has shown it not to be psychometrically valid in its native form. ${ }^{13}$ The Visual Function Index 14 (VF-14) questionnaire was originally developed to assess the functional impairment caused by cataract, ${ }^{14}$ but has since undergone preliminary validation for use in patients with $\mathrm{AMD},{ }^{15}$ specifically neovascular AMD (nAMD), ${ }^{16}$ although not 


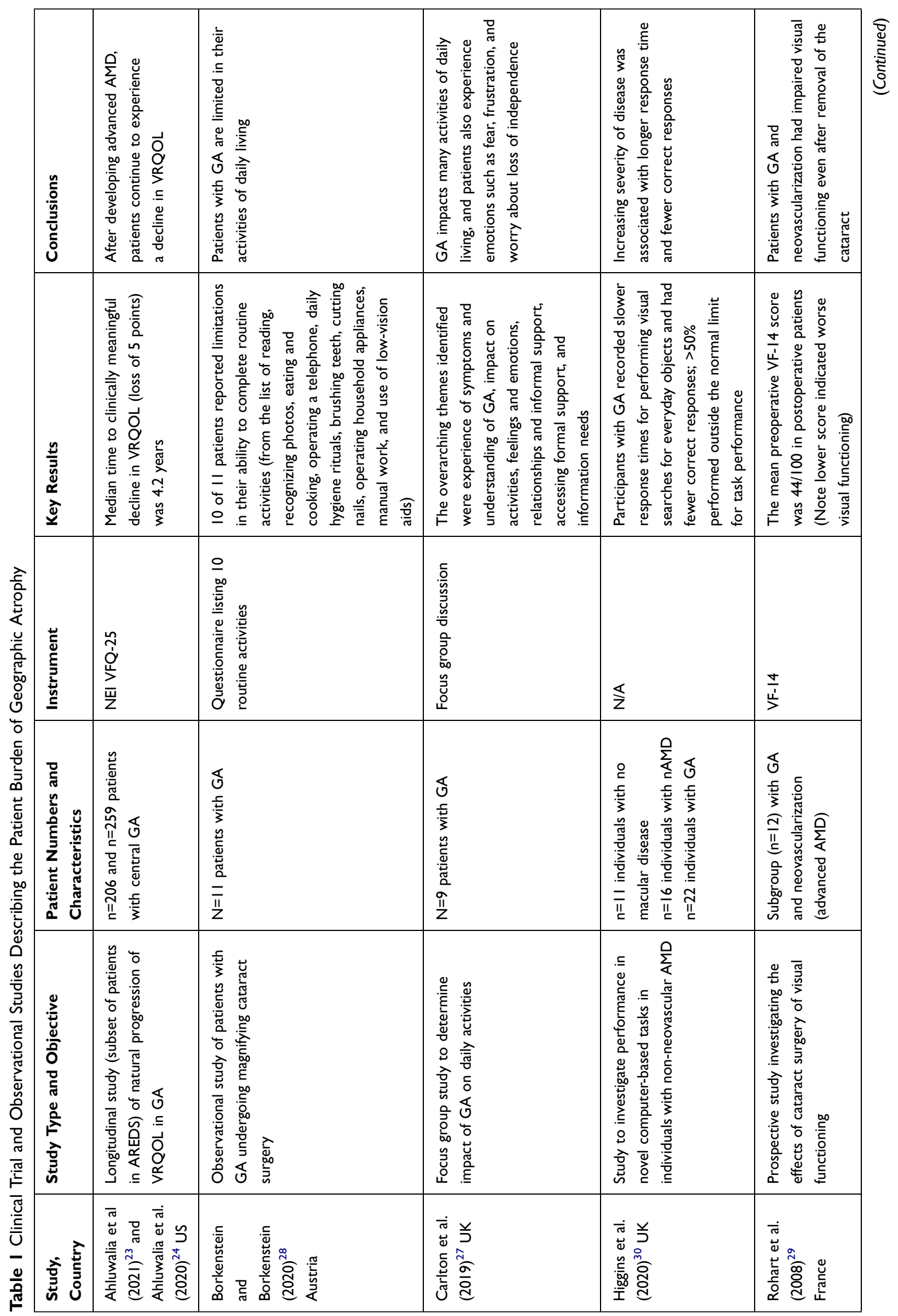




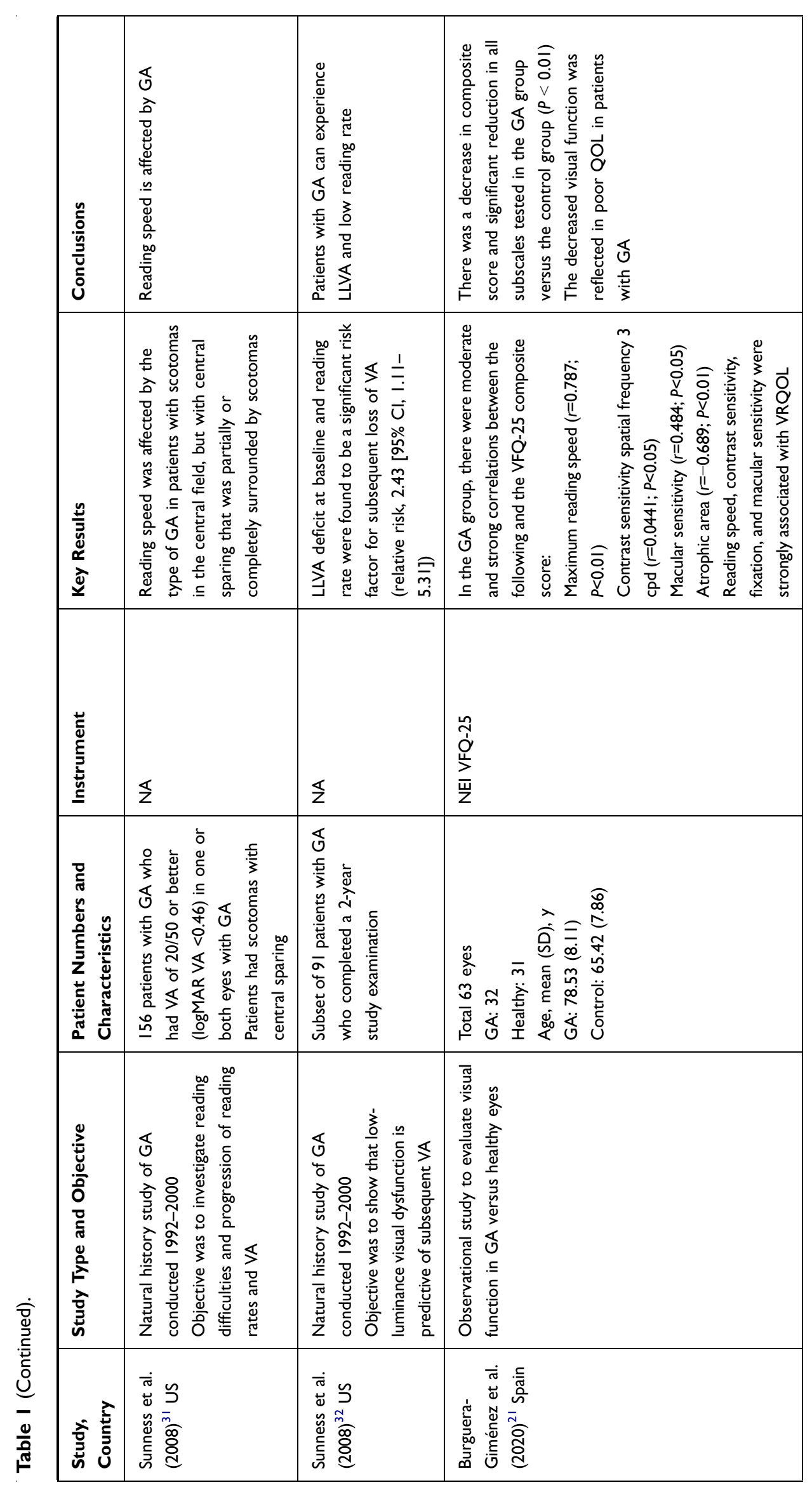




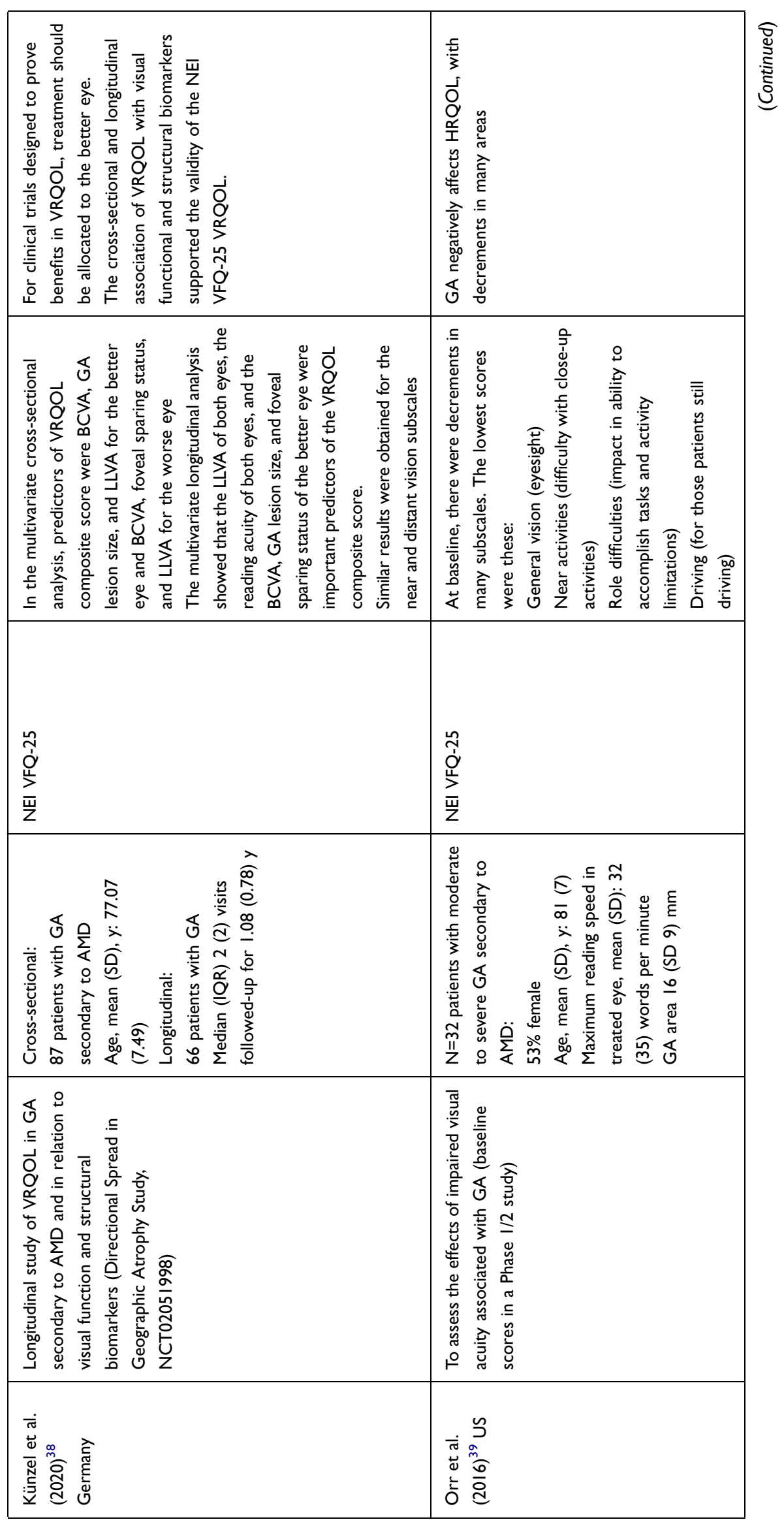




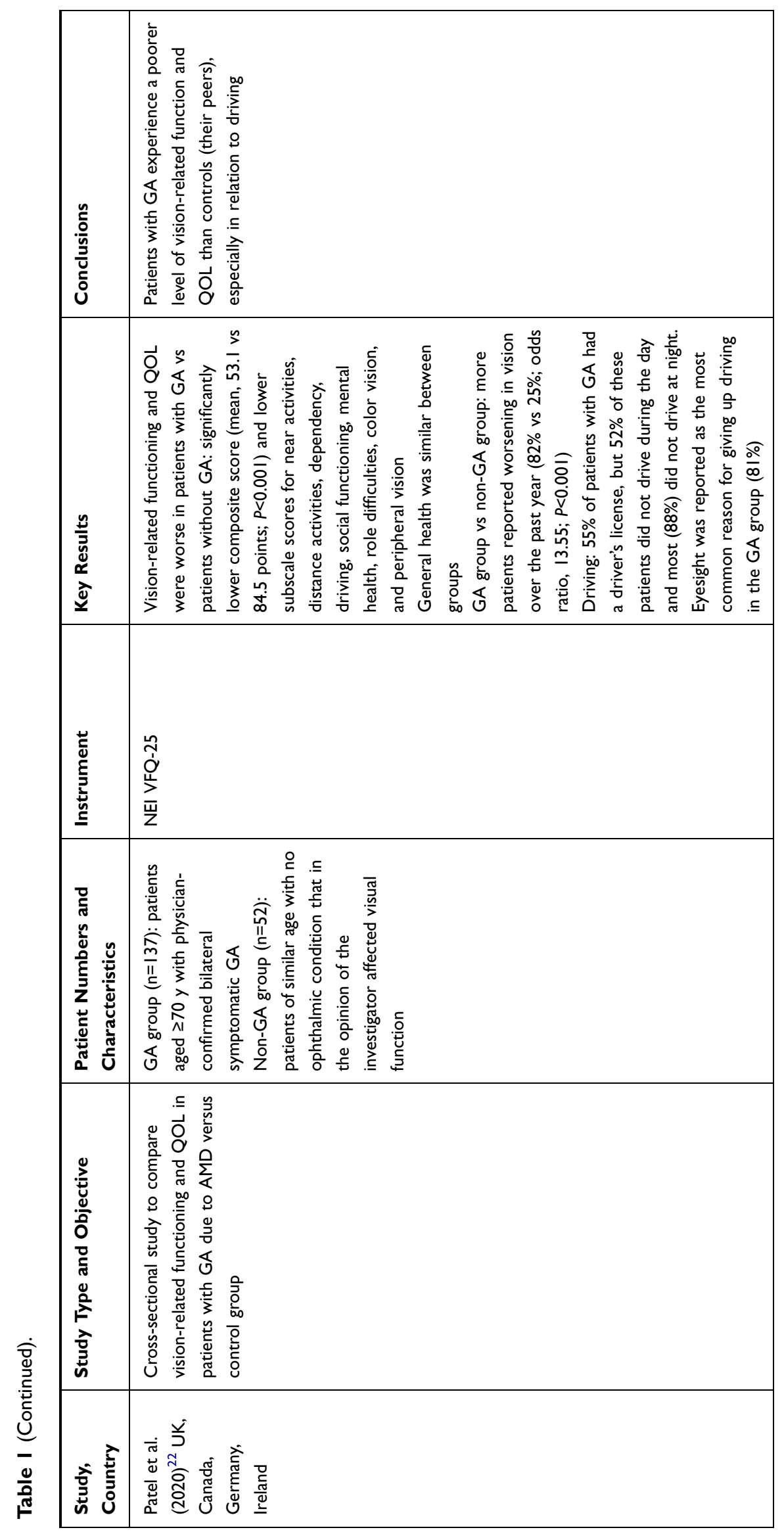




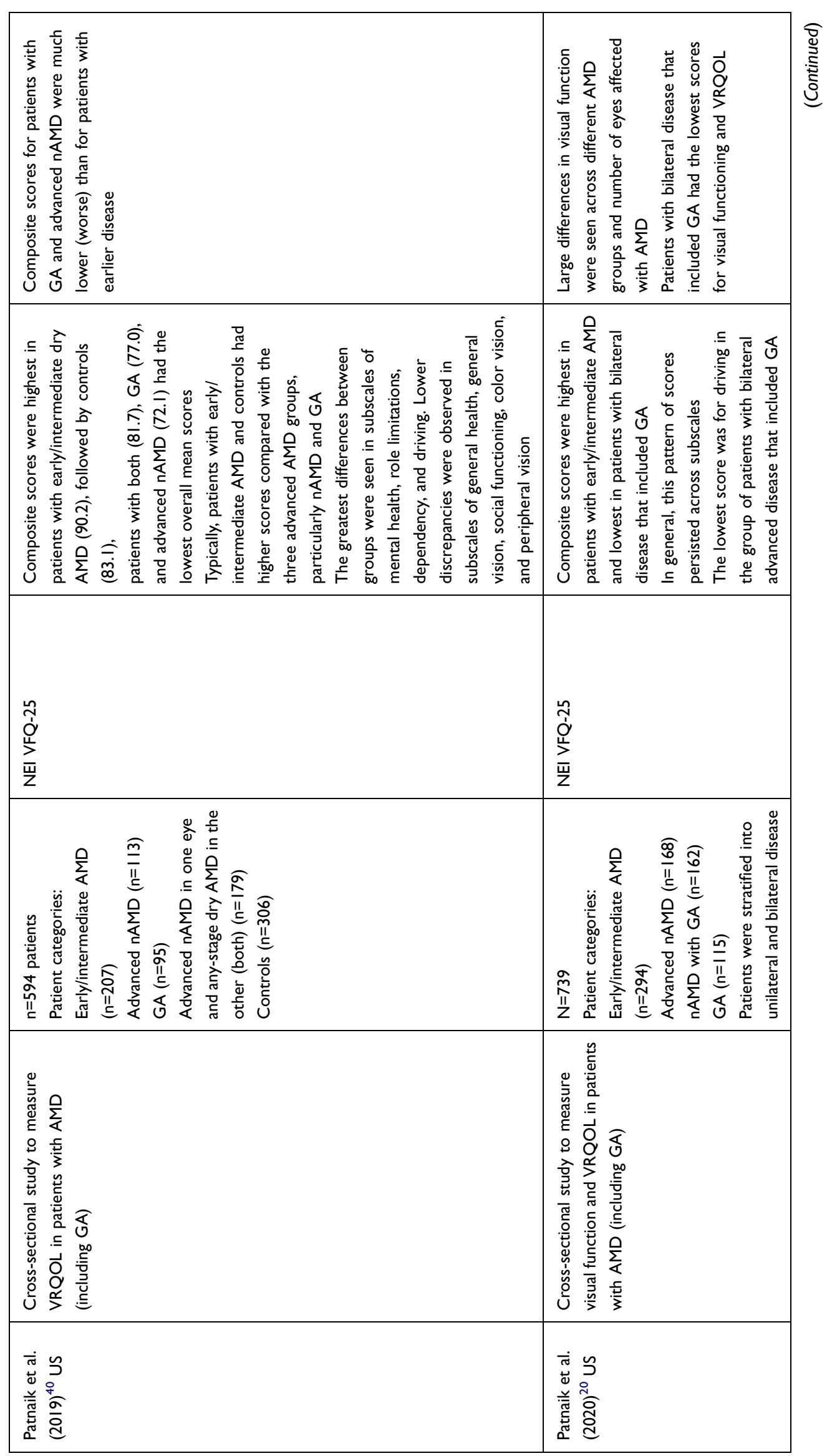




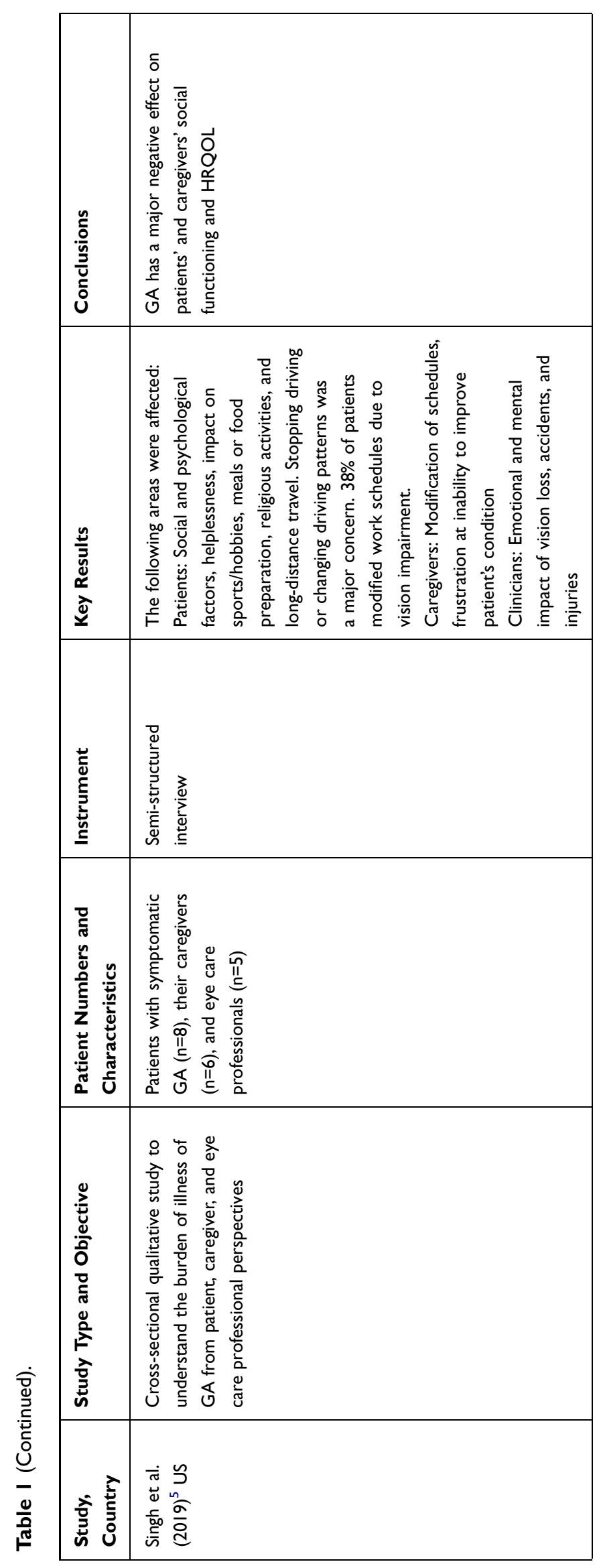




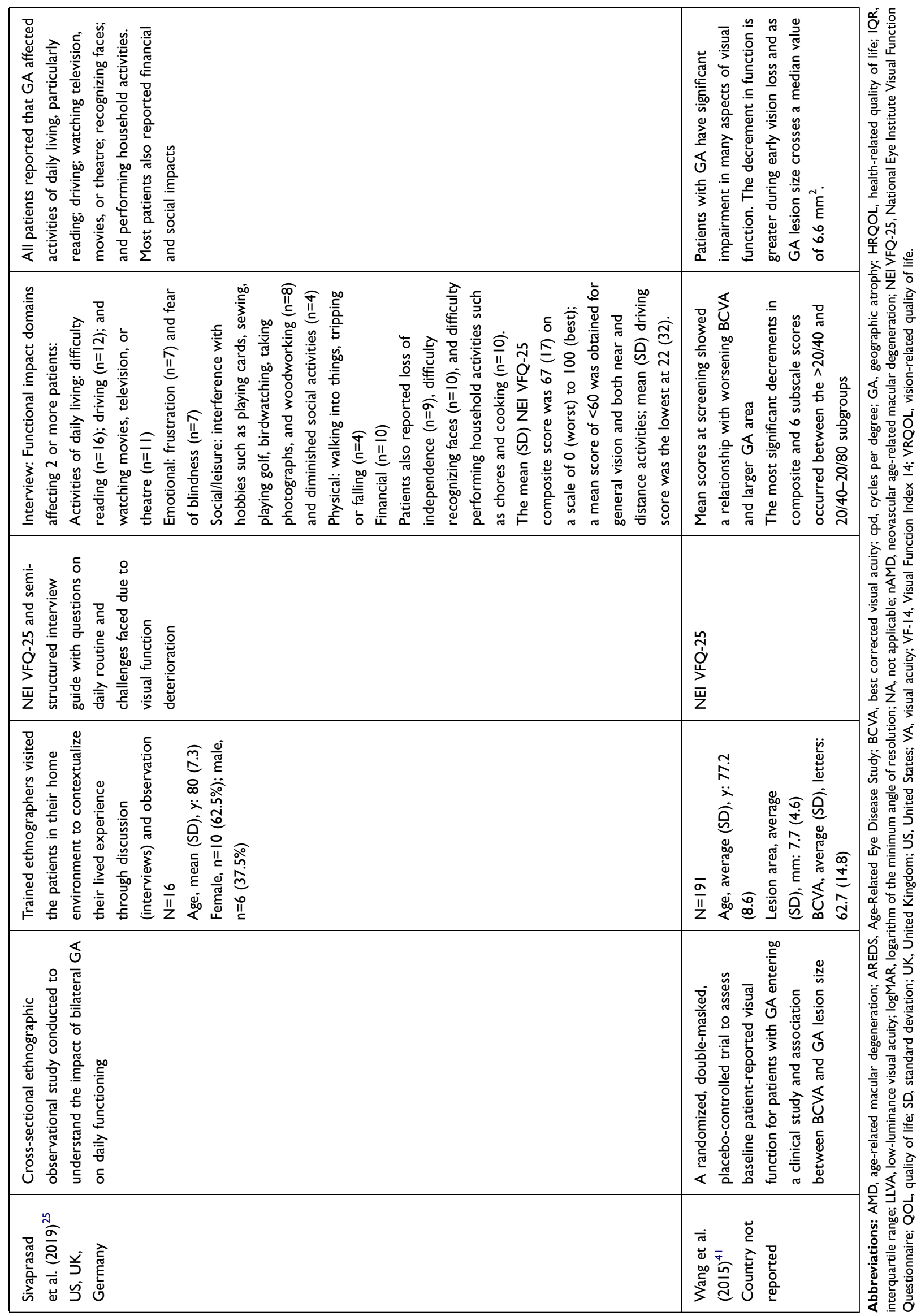


in patients with GA. The Functional Reading Independence (FRI) Index is a new seven-item instrument developed to assess the functional impact of GA. ${ }^{17}$

More broadly, in AMD, standardized measurement of outcomes, including measurement of vision-related quality of life (VRQOL), has been advocated by the International Consortium for Health Outcomes Measurement (ICHOM) working group. Specifically, the ICHOM has recommended that the brief Impact of Vision Impairment (IVI) questionnaire, which has appropriate content and reliability in patients with AMD (including those with late AMD) and has also undergone Rasch analysis and psychometric validation, should be used to assess visual functioning and VRQOL in patients with AMD. ${ }^{18,19}$

\section{Impact of GA on Vision- and Health-Related Quality of Life}

Several studies have evaluated VRQOL and health-related quality of life (HRQOL) in GA relative to other ocular conditions. Quality of life in patients with GA, as assessed by the NEI VFQ-25, was shown in a cross-sectional US study to be worse than that in patients with early/intermediate AMD and nAMD. ${ }^{20}$ A study of 739 patients with AMD showed that mean composite scores were highest among early/intermediate patients (89.9) and lowest for the groups with bilateral GA (71.3) and bilateral GA plus nAMD (68.5). Overall, patients with bilateral disease consistently had lower mean scores than their unilateral counterparts. In addition, an observational study in Spain evaluated visual function and VRQOL using the NEI VFQ-25 in 63 study eyes in 63 patients $\geq 50$ years old. Thirty-two eyes were identified as having GA and 31 eyes had normal macular health. For composite scores and all subscales, mean NEI VFQ-25 scores for the GA group were statistically significantly lower (worse) than that for the control group. ${ }^{21}$ A cross-sectional study comparing the HRQOL of patients with GA $(n=137)$ with patients of similar age with no ophthalmic conditions $(n=52)$ showed that GA is associated with a reduction in HRQOL. Patients in the GA group had a significantly lower mean (standard deviation [SD]) NEI VFQ-25 composite score than those in the non-GA group (53.1 [19.05] vs 84.5 [6.55] points, respectively; $P<0.001) .^{22}$

Geographic atrophy affects multiple aspects of VRQOL and HRQOL. A US cross-sectional qualitative study explored the burden of illness of GA from the perspectives of patients $(n=8)$, caregivers $(n=6)$, and eye care professionals $(\mathrm{n}=5) .{ }^{5}$ Topics mentioned most frequently by patients were driving, reading, and psychological issues. One-fourth of patients mentioned curtailing long-distance trips, 63\% reported difficulty reading for everyday tasks or leisure, and 38\% felt helpless or embarrassed that they required assistance. All patients reported that they still performed household chores, but they sometimes took longer to complete. Caregivers provided comments similar to those of patients, citing frustration at watching patients struggle. Caregivers provided transportation, assisted with household tasks, and offered help. Caregivers linked injuries to GA even when the patients did not. ${ }^{5}$ Eye care professionals reported that they routinely screened for psychological well-being, and suggested that patients in urban areas have less difficulty getting around due to public transportation, while patients in rural areas are more isolated. ${ }^{5}$

Evidence suggests that VRQOL in patients with GA declines over time as the disease progresses. The rate of decline in VRQOL was assessed using the NEI VFQ-25 in patients with central GA in the US AREDS study. ${ }^{23,24}$ A clinically meaningful decrease in VRQOL was defined as a loss of 5 points, and the median time to significant loss of VRQOL was 4.2 years (95\% CI, 3.0-5.7 years) in 259 patients, ${ }^{24}$ corresponding to a decline in $1.68 \pm 4.65$ NEI VFQ-25 units/year (107 patients). ${ }^{23}$

\section{Impact of GA on Functioning and Visual Performance}

Geographic atrophy has a profound effect on patient functioning and activities of daily living and can limit independence. Sivaprasad et $\mathrm{al}^{25}$ investigated the impact of GA on daily routine activities of 16 patients with GA in Germany, the United Kingdom (UK), and the US. Patients reported difficulties with reading (100\%), driving (75\%), and watching television, films, or theatre $(68.8 \%)$. Other concepts reported included loss of independence $(56.3 \%)$ and difficulty in recognizing faces $(62.5 \%)$ or performing household activities $(62.5 \%){ }^{25}$

A retrospective cohort analysis in the UK of 1901 patients with bilateral GA found that over time, $16 \%$ of patients became legally blind (median time to progression of 6.2 years [interquartile range (IQR), 3.3-8.5]) and $66.7 \%$ became ineligible to drive (median time to progression of 1.6 years [IQR, 0.7-2.7]), indicating the degree of the visual disability associated with GA. ${ }^{26}$ Results from a small focus group study of 9 UK patients with GA 
confirmed its impact on activities of daily living and reading. Visual symptoms such as blurring, distortion, and changes in perception affected activities such as driving, traveling as a passenger in a car, and walking; several participants had given up driving. ${ }^{27}$ Reading for both pleasure and necessity (eg, reading menus or card machines) was also impacted by GA. Other activities that participants had given up, or reported difficulties with, because of the symptoms of GA were housework, sewing and craft, puzzles, reading, music, and watching television. Furthermore, many of the participants worried about losing independence and becoming reliant on others.

Borkenstein and Borkenstein ${ }^{28}$ reported a study of 11 Austrian patients with GA who underwent magnifying cataract surgery using a foldable, bifocal high-add intraocular lens (IOL) in the better-seeing eye. When completing a questionnaire listing 10 routine activities (reading, recognizing photos, eating and cooking, operating a telephone, daily hygiene rituals, brushing teeth, cutting nails, operating household appliances, manual work, and use of low-vision aids [eg, magnifying glasses]) at 6 months postsurgery, 10 of 11 patients reported limitations in their ability to complete these activities.

A prospective French study of 12 eyes compared visual performance precataract and postcataract surgery; assessment included measurement of visual impairment using the VF-14 instrument. ${ }^{29}$ The study participants included a group $(\mathrm{n}=12)$ with GA and neovascularization (advanced AMD), among whom the mean postoperative VF-14 score was 44 of 100, indicating that the patients' visual performance was limited, even after removal of the cataract. $^{29}$

Higgins conducted a study to investigate performance in novel, computer-based tasks of everyday visual functioning in UK individuals with no macular disease $(\mathrm{n}=$ $11)$, nAMD $(n=16)$, or GA $(n=22)$. Participants with GA recorded slower response times than other groups for performing visual searches for everyday objects and had fewer correct responses; $>50 \%$ performed outside the normal limit for task performance. ${ }^{30}$ However, visual and reading acuity do not necessarily reflect functional reading ability in patients with GA and may depend on the location of atrophy. ${ }^{31,32}$ Sunness et $\mathrm{al}^{31}$ conducted a study of 156 US patients with GA who had visual acuity of 20/50 or better (logMAR visual acuity <0.46) in one or both eyes with GA to explore reading difficulties and the progression of reading impairment. Reading speed was affected by the pattern of GA, such that patients with scotomas in the central field, but with central sparing that is partially or completely surrounded by scotomas, had greater difficulty with larger letter sizes and reading from line to line because the words may not fit within the small central area that is surrounded by scotomas. ${ }^{31}$ Further, low reading rate was found to be a significant risk factor for subsequent loss of visual acuity (relative risk, 2.43 [95\% CI, 1.11-5.31]). ${ }^{32}$

\section{Depression and Visual Performance}

A US prospective cohort study of 51 older patients with recent-onset bilateral AMD evaluated Center for Epidemiological Studies Depression Scale (CES-D) scores, visual acuity, Functional Vision Screening Questionnaire scores, Chronic Disease Score, and the Community Disability Scale. ${ }^{1,33}$ Results showed that 17 patients $(33 \%)$ were depressed at baseline and had worse acuity $(P=0.04)$ and greater levels of vision-specific $(P=$ $0.03)$ and general $(P=0.002)$ physical disability than nondepressed patients. However, the correlations of CESD score with visual acuity and visual-specific disability were not significant after controlling for general physical disability. An increase in depressive symptoms over time predicted a decline in self-reported visual function independent of changes in visual acuity or medical status $(P<$ $0.05) .^{33}$

\section{Economic Burden}

\section{Direct Costs and Health Care Resource Use}

While limited evidence on the economic burden of disease is available, direct costs for patients with GA can be substantial. In a US retrospective study $(\mathrm{N}=14,421)$, mean total first-year cost postdiagnosis per patient with GA to the health-care system was US $\$ 11,672$ (Figure 1). ${ }^{34}$ Moreover, patients with GA are at risk of falls and fractures, which generate costs. Among all patients with GA, approximately $9 \%$ had a diagnosis of a fall or fracture during the follow-up period. The adjusted odds of developing fractures were significantly higher in patients with unilateral GA compared with those with unilateral early or intermediate AMD (odds ratio, 1.553 [95\% CI, 1.073-2.242]) after adjustment for baseline characteristics and comorbidities. ${ }^{4}$ In another US study with a prospective cohort design, Anastasopoulos et $\mathrm{al}^{35}$ explored the relationship between AMD and incident hip fractures in the Medicare population and found that Medicare patients with a code for atrophic $\mathrm{AMD}$ had an 11\% increased risk (odds ratio, 


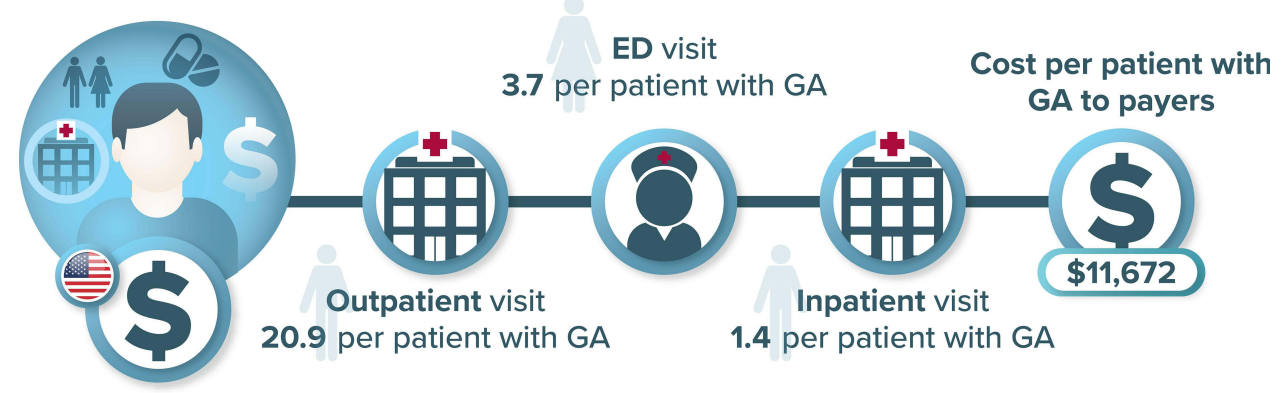

Figure I All-cause health care resource use (first year) by patients with GA in the United States.

Notes: Study captured all-cause RU and specific contributing factors to the economic burden of GA, and differentiators between severity levels were not explored. Costs have been adjusted to 2020 US dollars. Data from Kim A ${ }^{34}$

Abbreviations: ED, emergency department; GA, geographic atrophy; RU, resource use.

1.11 [95\% CI, 1.06-1.16]; $P<0.001)$ of hip fractures than patients without a code for AMD over a 4-year follow-up period.

Patients with GA incur variable resource use and costs, which are influenced by the presence of other ocular conditions; there is a lack of treatments and management strategies for GA. A retrospective analysis of data from medical records at different clinical sites in the UK was obtained to estimate per-patient direct ophthalmic resource use in patients with GA $(\mathrm{N}=$ 1080). ${ }^{3}$ Patients were divided into the following subgroups: GA in both eyes (GA:GA); GA in one eye, choroidal neovascularization (CNV) in the fellow eye (GA:CNV); and GA in one eye with early/intermediate AMD in the fellow eye (GA:E). Resource use was estimated for direct ophthalmology-related visits and associated costs (Figure 2). ${ }^{3}$ The most common monitoring test was visual acuity, followed by optical coherence tomography; both were most frequently performed in patients in the GA:CNV subgroup. This study did not include other direct costs such as the costs of antiVEGF therapies, and it is assumed that the higher costs associated with GA:CNV were due to more frequent monitoring of CNV. It was assumed that the monitoring of GA may have occurred for non-AMD-related pathology such as glaucoma, which was more common in the GA:GA and GA:E groups than in the GA:CNV group. ${ }^{3}$

In Europe, a retrospective chart review study investigated health-care resource use among patients in the UK, Germany, Ireland, and Canada with bilateral symptomatic GA versus a non-GA control group. ${ }^{22}$ The annualized total cost, consisting of direct ophthalmic health-care resource use, was $\$ 2115$ per patient with GA, mainly driven by diagnostic tests and procedures (\$1278 per patient) (Figure 3). The annualized average direct total cost per patient with GA in this study (\$2115) was comparable to direct vision-related medical costs reported for patients with neovascular AMD before anti-VEGF agents were approved

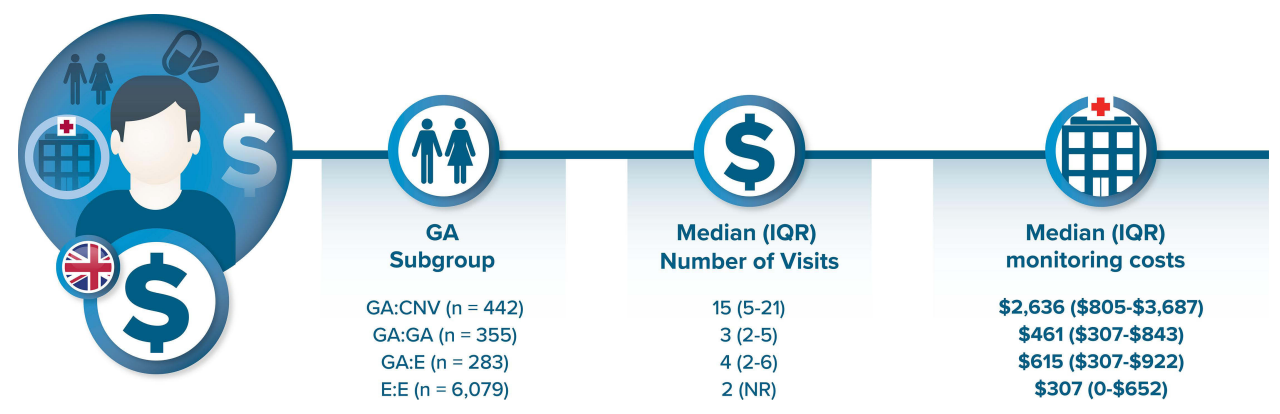

Figure 2 Median monitoring costs over 2 years across GA subgroups.

Notes: Patients with GA in the study eye and GA (GA:GA), choroidal neovascularization (GA:CNV), or early/intermediate AMD (GA:E) in the fellow eye, and patients with bilateral early/intermediate AMD (E:E). Costs applied per visit were \$153.63 (relating to Healthcare Resource Group service code I30: Ophthalmology; average unit cost) for a standard monitoring visit and $\$ 190.94$ (relating to Healthcare Resource Group cost code BZ88A; average unit cost) for a retinal tomography visit. Estimated median costs do not include treatment costs (eg, anti-VEGF plus injection). Patients were identified in the electronic medical record system from 10 clinical sites in the United Kingdom. Analyses were restricted to patients with the year of the index date being on or after January I, 20II, and who underwent $\geq 2$ years of follow-up. Costs have been adjusted to 2020 US dollars. Data from Chakravarthy et $\mathrm{al}^{3}$.

Abbreviations: GA, geographic atrophy; IQR, interquartile range; NR, not reported; VEGF, vascular endothelial growth factor. 


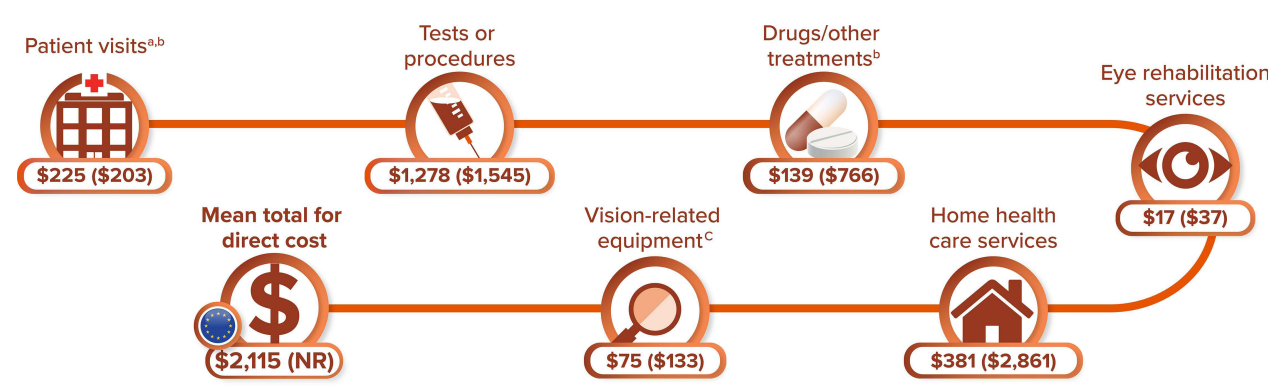

Figure 3 Medical and nonmedical resource utilization in the geographic atrophy group.

Notes: Mean (standard deviation) values are based on nonmissing values. ${ }^{a}$ For each patient, a cost was calculated for each test/procedure or treatment per period. Costs were adjusted if the patient had $<24$ months of history. Costs have been adjusted to 2020 US dollars. ${ }^{\text {b }}$ The unit cost per prescription was defined as the mean cost of all available prescriptions for each treatment, regardless of use. For Canada, a cost per pill was collected for amoxicillin, and because it is generally prescribed for depression with along duration of treatment, costs for its use were calculated for 6 months of treatment with aposology of one pill perday. ${ }^{\circ}$ Only one piece of equipment per period was considered to calculate the cost of vision-related equipment. Because the cost of reading aids was very different between the United Kingdom and Germany, the cost collected in the United Kingdom was applied to Germany. Data from Patel et al ${ }^{22}$.

Abbreviation: NR, not reported.

in the UK (\$3742). ${ }^{22,36}$ The total annual health-care cost for UK patients with AMD was $\$ 9214$; the cost of needing assistance with activities of daily living was the highest single contributor to the annual health resource utilization costs for patients with AMD [\$4434]. ${ }^{36}$ These studies suggest that GA is likely to be associated with large indirect costs, the extent of which is unclear given limited research on this topic.

\section{Indirect Costs}

To estimate the economic burden of visual loss from AMD in the US, Brown et $\mathrm{al}^{37}$ used data from the US Bureau of Labor and Statistics (accessed in 2004) that revealed that the employment rate was $30.6 \%$ for those with AMD and severe visual limitation (neovascular AMD, GA, or both). According to 1997 US mean wage data, a person with disabilities, such as mild visual loss and severe visual loss, earns 30\% (\$15,127 salary decrease) and 38\% less (\$19,124 salary decrease), respectively, than the mean wage. Assuming that $10 \%$ of the US Medicare population (aged $\geq 65$ years) have full-time employment and considering the prevalence of AMD in the US, the authors estimated a yearly loss to the US gross domestic product due to lost wages among people with severe vision loss due to GA or drusen $\geq 125 \mu \mathrm{m}$ to be approximately $\$ 39.4$ billion, not considering other costs such as transportation, caregivers, and injuries (Figure 4).

\section{Costs to Patients and Caregivers}

Patients and caregivers incur out-of-pocket costs; for US patients, these included vision aids such as magnifying systems, flashlights, or talking watches. Caregivers usually provided transportation and helped with household chores. ${ }^{5}$ A US retrospective cohort study of administrative claims data $(\mathrm{n}=14,421)$ from 2015 to 2018 determined the mean cost to the patient with GA to be $\$ 2863$ (adjusted to 2020 USD). However, no contributing factors to the burden of GA were described. ${ }^{34}$

\section{Discussion}

Geographic atrophy is a chronic, progressive condition with no effective treatment, resulting in a poor prognosis and significant unmet needs. ${ }^{10}$ Evidence related to the humanistic burden of GA consistently uses the NEI VFQ-25, which has undergone preliminary validation in patients with GA, although a minimum clinically important difference in score changes is not yet available. The humanistic burden of GA over time requires further research, and there are also gaps in the evidence characterizing the effect of vision loss on patient functioning. The FRI Index has been used to assess the impact of GA on functional reading independence, demonstrating good reliability and validity in a small study in patients with GA $(\mathrm{N}=100){ }^{17}$ further validation work will be required. In addition, very little information was identified on the humanistic effect on caregivers.

There are gaps in the evidence documenting the economic burden of GA. Our review identified only 7 studies with heterogeneous methodologies reporting on resource utilization and costs associated with GA, and no economic models. We identified only one study, published in 2005, on indirect costs. The need for more evidence on productivity impacts and indirect costs of GA is of particular importance because the societal costs of the disease are currently not well understood and could be significant. ${ }^{37}$ 


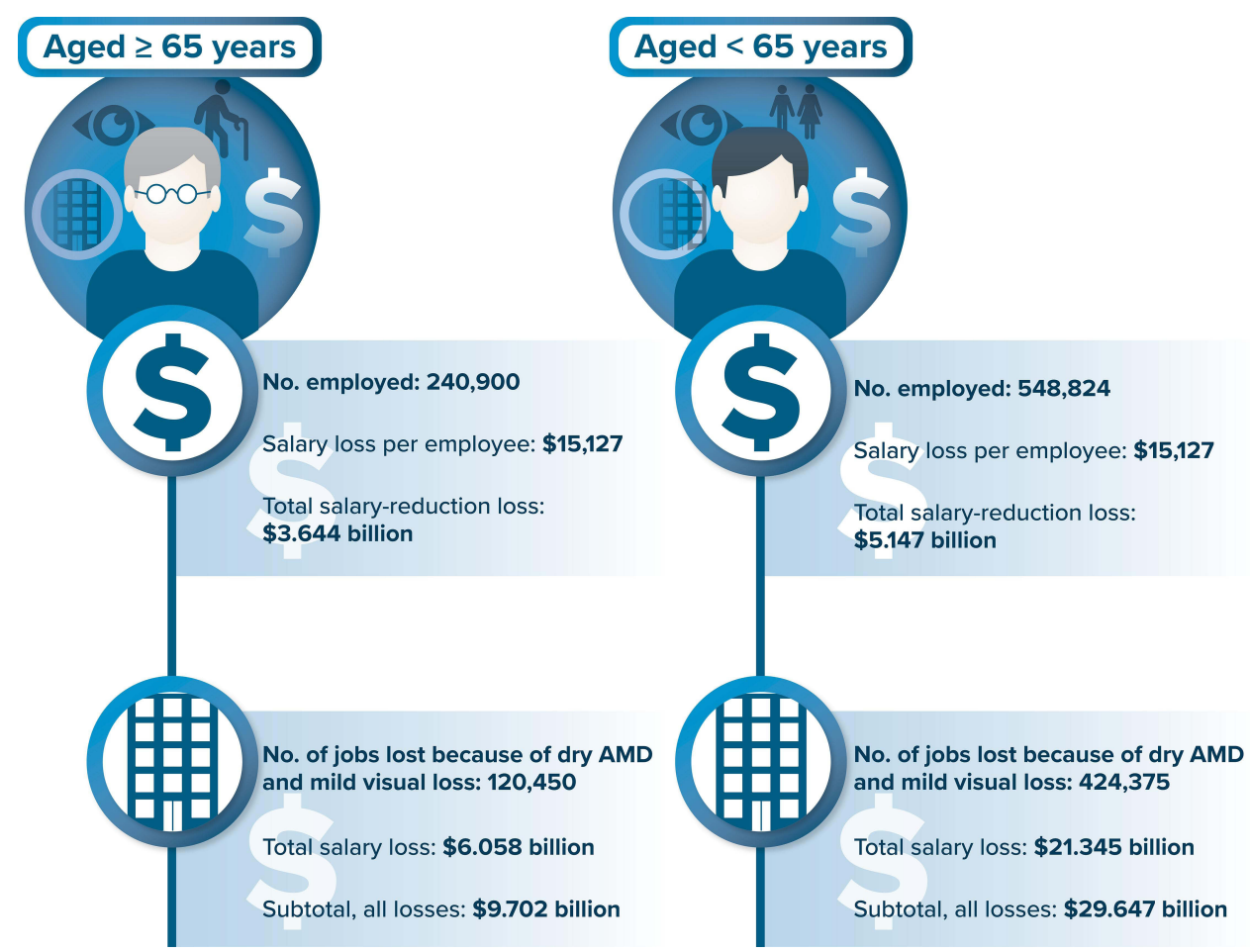

Figure 4 Annual loss to the US gross domestic product due to GA or drusen $\geq 125 \mu \mathrm{m}$ in diameter.

Notes: Details on dry AMD not specified in the article. Results are pooled for GA and dry AMD with drusen $\geq 125 \mu \mathrm{m}$ and thus should be interpreted with caution. Costs have been adjusted to 2020 US dollars. Data from Brown et $\mathrm{al}^{37}$.

Abbreviations: AMD, age-related macular degeneration; GA, geographic atrophy; US, United States.

Further, assuming that the incidence of GA increases with age, current-day indirect costs are likely to exceed the estimates in the available published evidence.

\section{Conclusion}

In conclusion, GA represents a significant unmet need. There currently are no effective treatments, and the humanistic burden, economic burden, and unmet needs are not well understood. Interventions that reduce the disability caused by GA would potentially preserve patients' and caregivers' quality of life and could also reduce indirect costs associated with poor vision and blindness. Future research to determine the true economic burden of GA would enable payers to make more robust decisions regarding the value of future treatment options.

\section{Abbreviations}

AMD, age-related macular degeneration; AREDS, AgeRelated Eye Disease Study; CES-D, Center for Epidemiological Studies Depression Scale; CI, confidence interval; CNV, choroidal neovascularization; FRI, Functional Reading Independence; GA, geographic atrophy; HRQOL, health-related quality of life; IQR, interquartile range; logMAR, logarithm of the minimum angle of resolution; nAMD, neovascular age-related macular degeneration; NEI VFQ-25, National Eye Institute Visual Function Questionnaire; SD, standard deviation; UK, United Kingdom; US, United States; VEGF, vascular endothelial growth factor; VF-14, Visual Function Index 14; VRQOL, vision-related quality of life.

\section{Acknowledgments}

Kate Lothman of RTI Health Solutions provided medical writing services during development of this manuscript. These services were funded by Apellis Pharmaceuticals. This study was conducted under a research contract between RTI Health Solutions and Apellis Pharmaceuticals and was funded by Apellis Pharmaceuticals.

\section{Disclosure}

SPS, WC, MI, and DLJ are employees of Apellis. AH, MB, and TT are employees of RTI Health Solutions. SW was an employee of RTI Health Solutions when this research was conducted. The author reports no other conflicts of interest in this work. 


\section{References}

1. Sacconi R, Corbelli E, Querques L, Bandello F, Querques G. A review of current and future management of geographic atrophy. Ophthalmol Ther. 2017;6(1):69-77. doi:10.1007/s40123-017-0086-6

2. Fleckenstein M, Mitchell P, Freund KB, et al. The progression of geographic atrophy secondary to age-related macular degeneration. Ophthalmology. 2018;125(3):369-390. doi:10.1016/j.ophtha.2017. 08.038

3. Chakravarthy U, Bailey CC, Scanlon PH, et al. Direct ophthalmic healthcare resource use among patients with geographic atrophy in a large cohort from the United Kingdom. Ophthalmol Retina. 2019;3 (11):920-926. doi:10.1016/j.oret.2019.06.012

4. Kim A, Shirneshan E, Campbell J, Devine B, Bansal A. Characterizing the healthcare resource utilization and costs in patients with geographic atrophy secondary to age-related macular degeneration stratified by disease severity (poster). Presented at the Virtual International Meeting of the International Society for Pharmacoeconomics and Outcomes Research; May 18-20; 2020.

5. Singh RP, Patel SS, Nielsen JS, Schmier JK, Rajput Y. Patient-, caregiver-, and eye care professional-reported burden of geographic atrophy secondary to age-related macular degeneration. $\mathrm{Am}$ $J$ Ophthalmic Clin Trials. 2019;2(1):1-6. doi:10.25259/AJOCT9-2018

6. Rudnicka AR, Kapetanakis VV, Jarrar Z, et al. Incidence of late-stage age-related macular degeneration in American whites: systematic review and meta-analysis. Am J Ophthalmol. 2015;160(1):85-93.e3. doi:10.1016/j.ajo.2015.04.003

7. Lum F, Repka MX, Vicchrilli S. How to use the ICD-10 codes for age-related macular degeneration. EyeNet Magazine; 2017.

8. Wright CB, Ambati J. Dry age-related macular degeneration pharmacology. Handb Exp Pharmacol. 2017;242:321-336.

9. Nebbioso M, Lambiase A, Cerini A, Limoli PG, La Cava M, Greco A. Therapeutic approaches with intravitreal injections in geographic atrophy secondary to age-related macular degeneration: current drugs and potential molecules. Int J Mol Sci. 2019;20(7):1693. doi:10.3390/ijms20071693

10. Nielsen MK. Geographic atrophy. American Academy of Ophthalmology. EyeWiki; October 1, 2020. Available from: https:// eyewiki.aao.org/Geographic_atrophy. Accessed October 19, 2020.

11. OECD. Organization of economic co-operation and development; 2021. Available from: https://www.oecd.org/. Accessed November 15, 2021.

12. Sivaprasad S, Tschosik E, Kapre A, et al. Reliability and construct validity of the NEI VFQ-25 in a subset of patients with geographic atrophy from the Phase 2 Mahalo Study. Am $J$ Ophthalmol. 2018;190:1-8. doi:10.1016/j.ajo.2018.03.006

13. Marella M, Pesudovs K, Keeffe JE, O'Connor PM, Rees G, Lamoureux EL. The psychometric validity of the NEI VFQ-25 for use in a low-vision population. Invest Ophthalmol Vis Sci. 2010;51 (6):2878-2884. doi:10.1167/iovs.09-4494

14. Steinberg EP, Tielsch JM, Schein OD, et al. The VF-14. An index of functional impairment in patients with cataract. Arch Ophthalmol. 1994;112(5):630-638. doi:10.1001/archopht.1994.01090170074026

15. Mackenzie PJ, Chang TS, Scott IU, et al. Assessment of vision-related function in patients with age-related macular degeneration. Ophthalmology. 2002;109(4):720-729. doi:10.1016/ S0161-6420(01)01021-1

16. Riusala A, Sarna S, Immonen I. Visual function index (VF-14) in exudative age-related macular degeneration of long duration. $\mathrm{Am}$ J Ophthalmol. 2003;135(2):206-212. doi:10.1016/S0002-9394(02) 01832-9

17. Kimel M, Leidy NK, Tschosik E, et al. Functional Reading Independence (FRI) Index: a new patient-reported outcome measure for patients with geographic atrophy. Invest Ophthalmol Vis Sci. 2016;57(14):6298-6304. doi:10.1167/iovs.16-20361
18. Mehta H, Tufail A, Daien V, et al. Real-world outcomes in patients with neovascular age-related macular degeneration treated with intravitreal vascular endothelial growth factor inhibitors. Prog Retin Eye Res. 2018;65:127-146. doi:10.1016/j.preteyeres.2017.12.002

19. Rodrigues IA, Sprinkhuizen SM, Barthelmes D, et al. Defining a minimum set of standardized patient-centered outcome measures for macular degeneration. Am J Ophthalmol. 2016;168:1-12. doi:10.1016/j.ajo.2016.04.012

20. Patnaik JL, Lynch AM, Pecen PE, et al. The impact of advanced agerelated macular degeneration on the National Eye Institute's Visual Function Questionnaire-25. Acta Ophthalmol. 2020. doi:10.1111/ aos. 14731

21. Burguera-Giménez N, García-Lázaro S, España-Gregori E, et al. Multimodal evaluation of visual function in geographic atrophy versus normal eyes. Clin Ophthalmol. 2020;14:1533-1545. doi:10.2147/ OPTH.S246245

22. Patel PJ, Ziemssen F, Ng E, et al. Burden of illness in geographic atrophy: a study of vision-related quality of life and health care resource use. Clin Ophthalmol. 2020;14:15-28. doi:10.2147/OPTH. S226425

23. Ahluwalia A, Shen LL, Del Priore LV. Central geographic atrophy vs. neovascular age-related macular degeneration: differences in longitudinal vision-related quality of life. Graefes Arch Clin Exp Ophthalmol. 2021;259(2):307-316. doi:10.1007/s00417-020-04892-5

24. Ahluwalia A, Shen LL, Del Priore LV. Natural history of vision-related quality of life in advanced age-related macular degeneration. Invest Ophthalmol Vis Sci. 2020;61(7):1937.

25. Sivaprasad S, Tschosik EA, Guymer RH, et al. Living with geographic atrophy: an ethnographic study. Ophthalmol Ther. 2019;8 (1):115-124. doi:10.1007/s40123-019-0160-3

26. Chakravarthy U, Bailey CC, Johnston RL, et al. Characterizing disease burden and progression of geographic atrophy secondary to age-related macular degeneration. Ophthalmology. 2018;125(6):8 42-849. doi:10.1016/j.ophtha.2017.11.036

27. Carlton J, Barnes S, Haywood A. Patient perspectives in geographic atrophy (GA): exploratory qualitative research to understand the impact of GA for patients and their families. Br Ir Orthopt $J$. 2019;15(1):133-141. doi:10.22599/bioj.137

28. Borkenstein AF, Borkenstein EM. Four years of observation to evaluate autonomy and quality of life after implantation of a high-add intraocular lens in age-related macular degeneration patients. Case Rep Ophthalmol. 2020;11(2):448-456. doi:10.1159/000508914

29. Rohart C, Fajnkuchen F, Nghiem-Buffet S, Abitbol O, Badelon I, Chaine G. [Cataract surgery and age-related maculopathy: benefits in terms of visual acuity and quality of life-a prospective study] Chirurgie de la cataracte et degenerescence maculaire liee a l'age: benefice en termes d'acuite visuelle et de qualite de vie-etude prospective. J Fr Ophtalmol. 2008;31(6 Pt 1):571-577. doi:10.1016/ s0181-5512(08)75457-3

30. Higgins BE, Taylor DJ, Bi W, Binns AM, Crabb DP. Novel computer-based assessments of everyday visual function in people with age-related macular degeneration. PLoS One. 2020;15(12): e0243578. doi:10.1371/journal.pone. 0243578

31. Sunness JS, Rubin GS, Zuckerbrod A, Applegate CA. Foveal-sparing scotomas in advanced dry age-related macular degeneration. $J$ Vis Impair Blind. 2008;102(10):600-610. doi:10.1177/0145482X081 0201004

32. Sunness JS, Rubin GS, Broman A, Applegate CA, Bressler NM, Hawkins BS. Low luminance visual dysfunction as a predictor of subsequent visual acuity loss from geographic atrophy in age-related macular degeneration. Ophthalmology. 2008;115(9):1480-8, 8 e1-2. doi:10.1016/j.ophtha.2008.03.009

33. Rovner BW, Casten RJ, Tasman WS. Effect of depression on vision function in age-related macular degeneration. Arch Ophthalmol. 2002;120(8):1041-1044. doi:10.1001/archopht.120.8.1041 
34. Kim A. Characterizing the healthcare resource utilization and costs by disease severity among patients with geographic atrophy secondary to age-related macular degeneration. ResearchWorks Archive; 2019. Available from: https://digital.lib.washington.edu/research works/handle/1773/44687. Accessed November 19, 2021.

35. Anastasopoulos E, Yu F, Coleman AL. Age-related macular degeneration is associated with an increased risk of hip fractures in the Medicare database. Am J Ophthalmol. 2006;142(6):1081-1083. doi:10.1016/j.ajo.2006.06.058

36. Lotery A, Xu X, Zlatava G, Loftus J. Burden of illness, visual impairment and health resource utilisation of patients with neovascular age-related macular degeneration: results from the UK cohort of a five-country cross-sectional study. $B r J$ Ophthalmol. 2007;91 (10):1303-1307. doi:10.1136/bjo.2007.116939

37. Brown MM, Brown GC, Stein JD, Roth Z, Campanella J, Beauchamp GR. Age-related macular degeneration: economic burden and value-based medicine analysis. Can J Ophthalmol. 2005;40 (3):277-287. doi:10.1016/S0008-4182(05)80070-5
38. Künzel SH, Möller PT, Lindner M, et al. Determinants of quality of life in geographic atrophy secondary to age-related macular degeneration. Invest Ophthalmol Vis Sci. 2020;61(5):63. doi:10. 1167/iovs.61.5.63

39. Orr SC, Pierson R, Bogert J, et al. Disease burden associated with geographic atrophy secondary to age-related macular degeneration. Invest Ophthalmol Vis Sci. 2016;57(12):21.

40. Patnaik J, Pecen P, Lynch A, Siringo F, Mathias M, Mandava N. The National Eye Institute Visual Function Questionnaire-25 in patients with age-related macular degeneration and controls. Invest Ophthalmol Vis Sci. 2019;60(9):67.

41. Wang F, Fries M, Wurzelmann JI, et al. Patient-reported visual function in patients with geographic atrophy secondary to age-related macular degeneration (AMD): baseline characteristics of the BAM114341 cohort. Invest Ophthalmol Vis Sci. 2015;56(7):2811. doi:10.1167/iovs.14-16009
Clinical Ophthalmology

\section{Publish your work in this journal}

Clinical Ophthalmology is an international, peer-reviewed journal covering all subspecialties within ophthalmology. Key topics include: Optometry; Visual science; Pharmacology and drug therapy in eye diseases; Basic Sciences; Primary and Secondary eye care; Patient Safety and Quality of Care Improvements. This journal is indexed on PubMed

\section{Dovepress}

Central and CAS, and is the official journal of The Society of Clinical Ophthalmology (SCO). The manuscript management system is completely online and includes a very quick and fair peer-review system, which is all easy to use. Visit http://www.dovepress.com/ testimonials.php to read real quotes from published authors. 